\title{
My first five years in science
}

\author{
Linus Pauling
}

\section{Linus Pauling, a giant of modern chemistry, dled on 19 August (see page 584 of last week's issue). What follows is an account, in his own words, of his first years as a research scientlst.}

SEVENTY-TWO years ago, in the spring of 1922 , I was completing my fourth year in the Oregon Agricultural College. In June 1922 I received the degree of Bachelor of Science in chemical engineering. I had learned a little mathematics, physics and a moderate amount of engineering. In addition I had amassed a tremendous amount of information about the properties of substances, especially inorganic compounds, including minerals and alloys. I had become interested in the electron theory of valence in 1919, and I continued to hope that the empirical information about the properties of substances could eventually be encompassed in a theory of the structure of molecules.

I had also applied to several universities for work, and had accepted an appointment in the California Institute of Technology (Caltech). My introduction to research began in September 1922, when I arrived in Pasadena, and I consider that my education continued for 5 years, until 1927, when I returned from Europe.

During my three and a half years as a graduate student I had learned a great deal about modern physics, about mathematics, and about chemistry, especially chemical thermodynamics (which did not appeal to me) and statistical mechanics, which I liked very much and still like. Caltech was a remarkable place. The first $\mathrm{PhD}$ had been given in 1920 , only 2 years before my arrival. The faculty contained many outstanding researchers in mathematics, physics and chemistry, including Roscoe Gilkey Dickinson (a pioneer X-ray crystallographer, the first recipient of a Caltech $\mathrm{PhD}$ ), Harry Bateman, Paul Epstein, Robert A. Millikan, Arthur Amos Noyes and Richard C. Tolman. There was great interest in line spectroscopy, the old quantum theory, and other aspects of modern physics. In the chemistry department, led by Noyes, graduate students were expected to begin research immediately on their arrival in Pasadena, and in addition to study some advanced courses, many taught by Tolman. I especially enjoyed Tolman's course in statistical mechanics.

In addition, there was a weekly seminar in chemistry, a seminar in physics, and a seminar held at either the Caltech campus or the Mount Wilson Observatory Building in Pasadena by the physics and astronomy club. All these seminars involved the presentation of striking, newly published discoveries, such as the Compton effect, the Stern-Gerlach experiment showing orientation of angularmomentum vectors in space, or the spin of the electron. My interest in X-ray crystallography has continued to the present. In addition, I attended courses of lectures by visiting physicists: Sommerfeld, Born (on matrix mechanics), Ehrenfest, Raman and Charles Galton Darwin. Although most of my early publications were based on experimental work, the determination of the structure of crystals, I soon began carrying out theoretical research.

I have had good luck several times during my life. One remarkable bit of good luck was in having gone to Caltech for my graduate studies. Years later, after I had become well acquainted with many outstanding universities, I realized that there was no place in the world in 1922 that would have prepared me in a better way for my career as a scientist.

After three and a half years in Pasadena I was sent to Europe as a research associate of Caltech, an arrangement made by Noyes. I had also applied for a John Simon Guggenheim Memorial fellowship, and shortly after my wife and I had arrived in Munich, I heard that the fellowship had been granted. We remained in Europe, one year in Munich with Sommerfeld and shorter stays in Copenhagen and Zurich, as well as visits to other centres of research in modern physics, for a year and a half. I had been given an appointment as an assistant professor at Caltech, and we returned to Pasadena in September 1927.

My education had continued during this period, especially during the year in Sommerfeld's Institute of Theoretical Physics. Just as we arrived, in April 1926, Schrödinger had begun publishing his papers on wave mechanics, and Sommerfeld immediately began giving lectures on them. Several other seminars were also devoted to newly published papers in wave mechanics and other aspects of quantum mechanics. I immediately began thinking about my main interest, which was not the hydrogen atom or hydrogen molecules, but rather the question of the structure and properties of many-electron atoms and ions and of more complex molecules. My paper was published in Zeitschrift für Physik for 1927. It involved an interesting combination of old quantum theory and wave mechanics, published by Gregor Wentzel earlier in 1926. The treatment involved the use of an atomic model developed in 1920 by Schrödinger, who suggested that atoms and ions containing many electrons might be treated by an idealized model in which the inner shells of electrons were replaced by surface charges of electricity, with a single electron in an orbit penetrating these shells. Wentzel used Sommerfeld's oldquantum-theory method of quantizing conditionally periodic systems, with the replacement of the square of the orbitalangular-momentum quantum number by the product of the quantum number and the quantum number plus 1 . Wentzel had evaluated the screening constants for $\mathrm{X}$ ray doublets by this theoretical treatment, but his calculated values disagreed with the experimental ones. I noticed that an extension of Wentzel's method could be carried out which led to reasonably good agreement with experiment. So far as I know, this was the first application of quantum mechanics to atoms and ions containing many electrons.

I then continued to develop this method and applied it to the calculation of theoretical values of the electric polarizability, diamagnetic susceptibility, extension in space of many-electron atoms and ions, and also to the evaluation of a set of values of radii of ions for use in ionic crystals. These papers were published in 1927.

The fact that I was in Sommerfeld's institute just at the time that Schrödinger published his papers on wave mechanics provides a second example of my astonishing good luck. So far as I know, Sommerfeld's lectures on wave mechanics were the first on this subject anywhere in the world. It was somewhat by chance that I arrived in Munich in the spring of 1926; I had in fact thought seriously of going to Niels Bohr's institute in Copenhagen, but circumstances caused me to change my mind. I am confident that this bit of good luck was largely responsible for my having got a rather thorough grounding in wave mechanics within a few months of its discovery, and probably responsible for my later success in applying quantum mechanics to some previously puzzling questions in the field of chemistry, culminating in the publication of my book, The Nature of the Chemical Bond, in 1939.

There have been other times in my life when I have had good luck and some when I have been less fortunate; but I see no reason to complain. I look back on the 5 years beginning in September 1922 with much pleasure, and with great gratitude to my remarkable teachers. 